DEBRIS DISKS AROUND SOLAR-TYPE STARS: OBSERVATIONS OF THE PLEIADES WITH THE SPITZER SPACE TELESCOPE

This article has been downloaded from IOPscience. Please scroll down to see the full text article. 2010 ApJ 7121421

(http://iopscience.iop.org/0004-637X/712/2/1421)

The Table of Contents and more related content is available

Download details:

IP Address: 131.215.193.213

The article was downloaded on 26/03/2010 at 21:27

Please note that terms and conditions apply. 


\title{
DEBRIS DISKS AROUND SOLAR-TYPE STARS: OBSERVATIONS OF THE PLEIADES WITH THE SPITZER SPACE TELESCOPE
}

\author{
J. M. Sierchio ${ }^{1}$, G. H. Rieke ${ }^{1}$, K. Y. L. Su ${ }^{1}$, P. Plavchan ${ }^{2}$, J. R. Stauffer ${ }^{3}$, And N. I. Gorlova \\ ${ }^{1}$ Steward Observatory, University of Arizona, Tucson, AZ 85721, USA; jsierchi@email.arizona.edu \\ ${ }^{2}$ Infrared Processing and Analysis Center, California Institute of Technology, MC 100-22, Pasadena, CA 91125, USA \\ ${ }^{3}$ Spitzer Science Center, California Institute of Technology, MC 103-33, Pasadena, CA 91125, USA \\ ${ }^{4}$ Instituut voor Sterrenkunde, K. U. Leuven, Celestijnenlaan 200D, 3001 Leuven, Belgium \\ Received 2009 November 16; accepted 2010 February 17; published 2010 March 15
}

\begin{abstract}
We present Spitzer MIPS observations at $24 \mu \mathrm{m}$ of 37 solar-type stars in the Pleiades and combine them with previous observations to obtain a sample of 71 stars. We report that 23 stars, or $32 \% \pm 6.8 \%$, have excesses at $24 \mu \mathrm{m}$ at least $10 \%$ above their photospheric emission. We compare our results with studies of debris disks in other open clusters and with a study of A stars to show that debris disks around solar-type stars at 115 Myr occur at nearly the same rate as around A-type stars. We analyze the effects of binarity and X-ray activity on the excess flux. Stars with warm excesses tend not to be in equal-mass binary systems, possibly due to clearing of planetesimals by binary companions in similar orbits. We find that the apparent anti-correlations in the incidence of excess and both the rate of stellar rotation and also the level of activity as judged by X-ray emission are statistically weak.
\end{abstract}

Key words: circumstellar matter - infrared: stars - open clusters and associations: individual (Pleiades) - stars: solar-type - stars: winds, outflows

Online-only material: color figures

\section{INTRODUCTION}

Understanding the evolution of planetary systems has long been central to our speculations about our place in the universe and is a challenging and rapidly advancing topic in astronomy and planetary science. Several hundred extrasolar planets have been discovered in the last 15 years, establishing that planetary systems form frequently. We have also significantly expanded our understanding of the planet formation process, through measurements of protoplanetary disks in the critical mid-infrared through submillimeter regimes (e.g., Andrews \& Williams 2005; Silverstone et al. 2006; Hernández et al. 2006) and development of detailed two- and three-dimensional numerical models to simulate and interpret these results (e.g., Alexander 2008; Dullemond et al. 2007). However, after protoplanetary disks dissipate at about 5 million years, the processes leading to planet formation and its remaining signatures become almost invisible to most types of observation.

Planetary debris disks, resulting from collisional activity among planetesimals (Wyatt 2008), are a notable exception and thus are our best current means to characterize planet system evolution. Debris disks are readily detected through the infrared emission from dust particles, which are produced in collisional cascades initiated among larger bodies, e.g., through gravitational stirring by planets. The generation of dust can be further enhanced when the particles become small enough that non-gravitational forces become significant, leading in extreme cases to avalanches of particle generation (Grigorieva et al. 2007). The dust is cleared from the disk on timescales of a thousand to a million years, so it must be replenished. Thus, debris disks are an effective indirect means to probe the current level of collisions in planetary systems.

One application of debris disk studies is to characterize the collisional activity as planet systems age (e.g., Habing et al. 2001; Spangler et al. 2001). Previous work has shown that debris disks tend to decay with time (Rieke et al. 2005;
Siegler et al. 2007). The excellent sensitivity of the Multiband Imaging Photometer on Spitzer (MIPS; Rieke et al. 2004) and, more importantly, the accurate photometry it can deliver, have expanded such studies substantially (e.g., Rieke et al. 2005; Su et al. 2006; Gorlova et al. 2006; Siegler et al. 2007; Meyer et al. 2008; Trilling et al. 2008; Hillenbrand et al. 2008; Carpenter et al. 2009b; Balog et al. 2009). In addition to accurate infrared photometry, such studies depend on the accurate determination of stellar ages. Substantial uncertainties remain in even the best age determinations for field stars (e.g., Mamajek \& Hillenbrand 2008). Therefore, measurements of excesses in stellar clusters, where ages are better determined, can refine the estimates of decay trends. However, the number of clusters close enough for the measurements to probe to solar masses and below is limited, and characterizing the decay trend and other disk parameters is therefore limited by poor statistics (Gáspár et al. 2009). For these reasons, it is important to observe thoroughly the small number of nearby rich clusters available. Only through such measurements can we test whether there are differences in disk behavior around different stellar types, with their accompanying differences in luminosities, masses, and presumably protoplanetary disk masses. For example, initial studies failed to find significant differences in infrared excess decay with respect to spectral type (e.g., Gorlova et al. 2006; Siegler et al. 2007), but with improved statistics they seem to be emerging (Gáspár et al. 2009). Removing age as a parameter also allows us to study other parameters affecting debris disk evolution, such as the various mechanisms for grain removal.

This paper completes our analysis of Spitzer debris disk surveys in the Pleiades. We report $24 \mu \mathrm{m}$ measurements of a sample of 37 stars of late $\mathrm{F}$ to early $\mathrm{K}$ type in this cluster. We combine these measurements with previous surveys by Stauffer et al. (2005) and Gorlova et al. (2006) to assemble a combined sample of 71 such stars. Our final excess rate provides the highest-weight determination available for debris disk behavior at $115 \pm 10 \mathrm{Myr}$, the age of the Pleiades (Meynet et al. 1993; 
Stauffer et al. 1998; Martín et al. 2001). In Section 2, we describe our observations and sample selection. In Section 3, we describe how we obtained excess ratios for our sample using both colorcolor plots and Kurucz model fitting. In Section 4, we interpret these excesses through comparisons with similar studies of Praesepe, Blanco 1, and NGC 2547. We also present an analysis of binarity, rotation, and stellar wind drag, as possible ways for the dust to be removed. Finally, in Section 5 we conclude with a summary and some possibilities for future work.

\section{OBSERVATIONS, DATA REDUCTION, AND SAMPLE SELECTION}

Table 1 lists our sample stars, with notations from Hertzsprung (HII: 1947), Artyukhina \& Kalinina (AK: 1970), and Pels et al. (1975, Van Leeuwen et al. 1986). Their Pleiades membership is based on criteria similar to those used by Stauffer et al. (2005), i.e., they are likely cluster members based upon radial velocities, proper motions, chromospheric and coronal activity indicators, and lithium abundance (Stauffer \& Hartmann 1987; Soderblom et al. 1993; Queloz et al. 1998; Rosvick et al. 1992). These stars are mostly of spectral types F5 to K1, when types are available; we also required $1.05<V-K_{S}<2.15$, appropriate for this range of types (Tokunaga 2000).

A few stars need to be discussed individually. HII 3031 is of indicated type F2 and fails the color selection. AK 1B 590 is indicated to be type F2 but passes the color test, so we have retained it. HII 1139 and Pels 128 were also rejected because the photometry may be influenced by close stars of similar brightness and HII 2172 was rejected because of a structured background. Thus, we report the photometry of HII 3031, HII 1139, HII 2172, Pels 128 , and Pels $173^{5}$, but do not include them in the analysis of the final sample. The remaining 32 objects are termed the new sample.

We used the MIPS in photometric mode to measure the $24 \mu \mathrm{m}$ emission from the stars in the new sample (PID: 30503) with final integration times of 93-313 s depending on the source brightness. The data were reduced with the MIPS Instrument Team Data Analysis Tool (DAT; Gordon et al. 2005) with the specific prescription for maximum photometric accuracy described by Engelbracht et al. (2007). The calibration is based on Rieke et al. (2008). We extracted photometry using pointspread function (PSF) fitting with a smoothed theoretical PSF generated by the STinyTim program (Krist 2006). We also compared the results for consistency with aperture photometry of the same star and examined each field for nearby objects or background structures that might compromise the photometry, leading to rejection of HII 1139, 2172, 3031, and Pels 128 mentioned above. Most of the stars reside far from the cluster center, reducing the risk of poorly measured or confused $24 \mu \mathrm{m}$ emission. The sample properties are summarized in Table 1.

We also include in our analysis other Pleiades members measured by Stauffer et al. (2005) and Gorlova et al. (2006).

\footnotetext{
5 Pels 173 was eliminated because of general confusion about its nature. It is generally considered to be the $\mathrm{C}$ component of the triple system,

$\mathrm{BD}+22617=\mathrm{HD} 25201$. The $\mathrm{A}$ and $\mathrm{B}$ components are a close B9/A binary with proper motion and photometric characteristics consistent with membership in the Pleiades, but with a parallax that puts them significantly behind the cluster. The $\mathrm{C}$ component, about $1^{\prime}$ removed, is of spectral type F2 and has photometric colors consistent with this designation, but is too faint by $\sim 0.6$ mag for an F2V star within the Pleiades. The most likely designation for these three stars is that they in fact are a physical system but are behind the Pleiades, and therefore, for our purposes, are not useful because there is no good age estimate. Many of the discussions of this star in the literature mix properties of the different components in non-physical combinations.
}

The full sample of Gorlova et al. (2006) includes Pleiades members of B type and later. We took from it all stars where the appropriate intrinsic colors matched the color selection in our new sample. Where spectral types are available, they generally agree with this color selection, although there are two stars within the color selection (HII 1338 and 1912) that are indicated to be F3 and F4, respectively. Although their relatively red colors for their types might arise from reddening, there might also be type errors, so we retained them in the sample. We rejected stars where the indicated noise exceeded $4 \%$ of the signal (HII 1095 , $1207,1794,2311,2341)$, since our goal is to detect excesses at this level $(1 \sigma)$. All together, the combined sample for analysis has 71 members, consisting of the 32 in our new sample observed for this paper plus all retained stars from Gorlova et al. (2006) and Stauffer et al. (2005). Table 2 lists this complete sample with flux measurements.

\section{DETERMINATION OF EXCESSES}

Identifying $24 \mu \mathrm{m}$ excesses requires an accurate extrapolation of the photospheric output to this wavelength. We define the ratio of the observed $24 \mu \mathrm{m}$ flux density to the extrapolated photospheric flux density, the excess ratio, as an indicator of the level of excess emission (no excess and no measurement errors would yield an excess ratio of unity). We first describe how we carried out this step for the new sample. We then discuss the steps to put the studies of Stauffer et al. (2005) and Gorlova et al. (2006) on the same scale to provide a uniform combined sample.

\subsection{The New Sample}

We assembled a database including available optical and near-infrared photometry for the stars in this sample. We used these data for two independent approaches to extrapolate the photospheric spectral energy distributions (SEDs), one based on fitting stellar SED models and the other based on applying photometric colors. Comparing the results of these methods shows that they give similar performance, so our final excess ratios are based on the average of the two SED extrapolations.

\subsubsection{SED Fitting}

The database listing for each star included, if found, spectral type, extinction, temperature, and the following photometry: Johnson $U B V R I$, Two Micron All Sky Survey (2MASS) $J H K_{S}$, Hipparcos $B V$, and Strömgen uvby. Almost all the stars had $B V R J H K_{S}$ photometry available, most had $U$ and $I$, and a few had the other bands. Table 1 presents our database of photometry, effective temperatures, surface gravities, spectral types, and metallicities used for SED fitting.

The SED fitting was based on Kurucz models (Castelli \& Kurucz 2004) and utilized an interactive code written in IDL. To create the SEDs, we used the constraint that the Kurucz modelfitting program fits the available photometry from 0.5 to $5 \mu \mathrm{m}$. We recorded the Hipparcos $B$ and $V$ photometry separately and included it in the fits as independent points from the literature $B V$ photometry, unless the literature photometry was based on those measurements. The effective temperatures listed in Table 1 were used as initial estimates in the SED fitting procedure. For each star we searched for models at $\pm 750 \mathrm{~K}$ centered near the expected effective temperature to obtain the best-fit SED. 
Table 1

Basic Properties of Pleiades Stars: New Sample

\begin{tabular}{|c|c|c|c|c|c|c|c|c|c|c|c|c|c|c|c|}
\hline Target & Other Name & S. T. ${ }^{\mathrm{a}}$ & $T_{\text {eff }}$ & $\log g^{b}$ & {$[\mathrm{Fe} / \mathrm{H}]^{\mathrm{c}}$} & $U$ & $B$ & $V$ & $R$ & $I$ & $J$ & $H$ & $K_{S}$ & AOR Key & References \\
\hline AK IA 36 & HIP 17317 & - & $5500^{\mathrm{d}}$ & - & - & - & 11.084 & 10.384 & 10.00 & $9.65^{\mathrm{e}}$ & 9.117 & 8.868 & 8.758 & 18304256 & $-,-,-,-,-, 1,1,2,3,4,4,4$ \\
\hline AK 1 A 56 & $\mathrm{BD}+21508$ & F8 & 6200 & - & - & - & 11.119 & 10.474 & 10.33 & - & 9.086 & 8.819 & 8.777 & 18304512 & $5,6,-,-,-, 7,7,8,-, 4,4,4$ \\
\hline AK 1 A 76 & HD 23312 & F5 & 6310 & 4.44 & - & 10.00 & 9.98 & 9.49 & 9.05 & 8.74 & 8.52 & 8.349 & 8.278 & 18306560 & $5,9,9,-, 10,10,10,10,10,4,4,4$ \\
\hline AK 1A 317 & HD 24463 & G0 & 6030 & - & - & - & 10.234 & 9.718 & 9.28 & - & 8.687 & 8.48 & 8.424 & 18303744 & $5,6,-,-,-, 1,1,2,-, 4,4,4$ \\
\hline AK 1B 7 & HD 22627 & G0 & $6000^{\mathrm{d}}$ & - & - & - & 10.417 & 9.831 & 9.47 & - & 8.728 & 8.54 & 8.434 & 18303488 & $11,-,-,-,-, 1,1,2,-, 4,4,4$ \\
\hline AK IB 8 & HIP 17044 & - & $5700^{\mathrm{d}}$ & - & - & - & 11.009 & 10.391 & 9.93 & $9.72^{\mathrm{e}}$ & 9.232 & 8.999 & 8.905 & 18306048 & $-,-,-,-,-, 1,1,8,3,4,4,4$ \\
\hline AK 1B 365 & HD 23598 & F8 & 6030 & - & - & - & 10.393 & 9.822 & 9.38 & - & 8.72 & 8.463 & 8.435 & 18304000 & $11,6,-,-,-, 12,12,2,-, 4,4,4$ \\
\hline AK 1B 590 & HD 24086 & $\mathrm{~F} 2$ & 6890 & - & - & - & 9.563 & 9.142 & 8.72 & - & 8.214 & 8.045 & 8.009 & 18302720 & $5,6,-,-,-, 7,7,8,-, 4,4,4$ \\
\hline AK 1B 560 & HD 23975 & G0 & 6462 & 4.16 & 0.06 & 10.17 & 10.16 & 9.64 & 9.2 & 8.87 & 8.645 & 8.469 & 8.382 & 18306816 & $5,13,13,13,10,10,10,10,10,4,4,4$ \\
\hline AK II 34 & HD 22444 & G0 & 6030 & - & - & 9.76 & 9.76 & 9.23 & 8.78 & 8.47 & 8.141 & 7.946 & 7.902 & 18305024 & $5,6,--,-10,10,10,10,10,4,4,4$ \\
\hline AK II 359 & TYC 1798-465-1 & - & $5000^{d}$ & - & - & - & 11.263 & 10.588 & 10.14 & - & 9.363 & 9.124 & 9.021 & 18304768 & $-,-,-,-,-, 7,7,8,-, 4,4,4$ \\
\hline AK II 383 & HIP 16979 & F8 & 6200 & - & - & - & 10.691 & 10.044 & 9.81 & $9.39^{\mathrm{e}}$ & 9.018 & 8.775 & 8.696 & 18305536 & $5,6,-,-,-, 1,1,8,3,4,4,4$ \\
\hline AK II 437 & HD 22680 & F8 & 6200 & - & - & - & 10.521 & 9.937 & 9.55 & $9.26^{\mathrm{e}}$ & 8.92 & 8.686 & 8.634 & 18305792 & $6,6,-,-,-, 7,7,8,3,4,4,4$ \\
\hline AK III 288 & HIP 16639 & F5 & 6440 & - & - & 10.06 & 10.05 & 9.54 & 9.06 & 8.74 & 8.553 & 8.312 & 8.274 & 18305280 & $5,6,-,-, 10,10,10,10,10,4,4,4$ \\
\hline HII 25 & HD 23061 & F5 & 6462 & 4.19 & -0.08 & 9.96 & 9.95 & 9.47 & 9.01 & 8.74 & 8.514 & 8.325 & 8.263 & 18311424 & $14,15,16,15,10,10,10,10,10,9,4,4,4$ \\
\hline HII 102 & TYC 1799-118-1 & G1 & $6100^{\mathrm{d}}$ & - & - & 11.39 & 11.24 & 10.51 & 9.90 & 9.44 & 9.101 & 8.722 & 8.655 & 18311680 & $14,-,-,-, 10,10,10,10,10,4,4,4$ \\
\hline HII 1132 & HD 23514 & G0 & 6545 & 4.21 & -0.02 & 9.95 & 9.92 & 9.42 & 8.96 & 8.67 & 8.479 & 8.291 & 8.153 & 18303232 & $11,15,13,15,10,10,10,10,10,4,4,4$ \\
\hline HII 1139 & HD 23513 & F5 & 6545 & 4.37 & 0.07 & 9.85 & 9.85 & 9.37 & 8.97 & 8.68 & 8.473 & 8.28 & 8.243 & 18311936 & $14,17,13,17,10,10,10,10,10,4,4,4$ \\
\hline HII 1766 & HD 23732 & F4 & 6720 & 4.5 & -0.02 & 9.67 & 9.60 & 9.13 & 8.70 & 8.41 & 8.137 & 7.912 & 7.862 & 18302976 & $14,18,18,18,19,19,19,19,19,4,4,4$ \\
\hline HII 2172 & HD 282965 & F9 & $6000^{\mathrm{d}}$ & - & - & 11.15 & 11.06 & 10.43 & 9.88 & 9.50 & 9.246 & 9.028 & 8.949 & 18312192 & $14,-,-,-, 10,10,10,10,10,4,4,4$ \\
\hline HII 3031 & HD 24132 & $\mathrm{~F} 2$ & 7000 & 3.98 & 0.09 & 9.3 & 9.25 & 8.87 & 8.49 & 8.27 & 8.059 & 7.93 & 7.877 & 18308096 & $14,13,13,13,19,19,19,19,19,4,4,4$ \\
\hline PELS 7 & TYC 1802-95-1 & - & $5600^{\mathrm{d}}$ & - & - & - & 11.142 & 10.379 & 10.08 & - & 9.24 & 8.953 & 8.833 & 18308352 & $-,-,-,-,-, 7,7,8,-, 4,4,4$ \\
\hline PELS 20 & HIP 17020 & - & $5600^{d}$ & - & - & - & 11.17 & 10.52 & 10.26 & $9.82^{\mathrm{e}}$ & 9.358 & 9.053 & 9.041 & 18308608 & $-,-,-,-,-, 1,1,8,3,4,4,4$ \\
\hline PELS 23 & HIP 17245 & - & $6000^{\mathrm{d}}$ & - & - & - & 10.673 & 10.102 & 9.72 & $9.44^{\mathrm{e}}$ & 8.959 & 8.657 & 8.593 & 18308864 & $-,-,-,-,-, 1,1,8,3,4,4,4$ \\
\hline PELS 25 & HIP 17125 & F5 & 6440 & - & - & - & 10.088 & 9.571 & 9.45 & $9.00^{\mathrm{e}}$ & 8.638 & 8.479 & 8.373 & 18309120 & $5,6,-,-,-, 7,7,8,3,4,4,4$ \\
\hline PELS 40 & $\mathrm{BD}+21516$ & - & $5700^{d}$ & - & - & - & 10.546 & 9.982 & 9.59 & - & 8.902 & 8.694 & 8.586 & 18307072 & $-,-,-,-,-, 7,7,2,-, 4,4,4$ \\
\hline PELS 86 & HIP 18544 & F8 & 6200 & - & - & - & 9.928 & 9.377 & 9.04 & $8.81^{\mathrm{e}}$ & 8.436 & 8.248 & 8.197 & 18309376 & $5,6,-,-,-, 1,1,2,3,4,4,4$ \\
\hline PELS 121 & $\mathrm{BD}+23455$ & - & $5700^{\mathrm{d}}$ & - & - & - & 10.891 & 10.294 & 10.03 & - & 9.066 & 8.754 & 8.679 & 18309632 & $5,-,-,-,-, 1,1,2,-, 4,4,4$ \\
\hline PELS 124 & HIP 16753 & - & $6100^{d}$ & - & - & - & 10.401 & 9.836 & 9.74 & $9.25^{\mathrm{e}}$ & 8.831 & 8.599 & 8.541 & 18307328 & ${ }_{-,-,-,-,-, 1,1,8,3,4,4,4}$ \\
\hline PELS 128 & BD+26 592 & G0 & 6030 & - & - & - & 11.012 & 10.276 & 9.91 & - & 9.003 & 8.778 & 8.66 & 18310144 & $5,6,-,-,-, 7,7,8,-, 4,4,4$ \\
\hline PELS 135 & TYC 1256-516-1 & F5 & 6440 & - & - & - & 9.869 & 9.391 & 8.91 & - & 8.404 & 8.225 & 8.152 & 18310400 & $6,6,-,-,-, 7,7,8,-, 4,4,4$ \\
\hline PELS 146 & HIP 18091 & - & $5600^{\mathrm{d}}$ & - & - & - & 11.17 & 10.513 & 9.91 & $9.80^{\mathrm{e}}$ & 9.267 & 8.981 & 8.873 & 18310656 & $-,-,-,-,, 20,20,8,3,4,4,4$ \\
\hline PELS 150 & HD 23935 & F8 & 6200 & - & - & 10.16 & 10.10 & 9.57 & 9.11 & 8.76 & 8.489 & 8.332 & 8.254 & 18310912 & $5,6,-,-, 10,10,10,10,10,4,4,4$ \\
\hline PELS $173^{f}$ & $\mathrm{BD}+22617 \mathrm{C}$ & - & $6500^{d}$ & - & - & - & 10.06 & 9.61 & - & - & 8.676 & 8.558 & 8.488 & 18311168 & $-,-,-,-,-, 21,21,-,-, 4,4,4$ \\
\hline PELS 174 & HIP 18955 & F5 & 6440 & - & - & - & 10.277 & 9.678 & 9.34 & $9.04^{\mathrm{e}}$ & 8.519 & 8.262 & 8.183 & 18307840 & $5,6,-,-,-, 1,1,8,3,4,4,4$ \\
\hline
\end{tabular}


Table 1

(Continued)

\begin{tabular}{|c|c|c|c|c|c|c|c|c|c|c|c|c|c|c|c|}
\hline Target & Other Name & S. T. ${ }^{\mathrm{a}}$ & $T_{\text {eff }}$ & $\log \mathrm{g}^{\mathrm{b}}$ & {$[\mathrm{Fe} / \mathrm{H}]^{\mathrm{c}}$} & $U$ & $B$ & V & $R$ & $I$ & $J$ & $H$ & $K_{S}$ & AOR Key & References \\
\hline $\operatorname{TrS} 42$ & HIP 17316 & G0 & 6030 & - & - & 10.44 & 10.41 & 9.86 & 9.38 & 9.07 & 8.797 & 8.604 & 8.526 & 18306304 & $5,6,-,-, 10,10,10,10,10,4,4,4$ \\
\hline $\operatorname{Tr} 60$ & HD 24302 & F8 & 6450 & 4.21 & -0.2 & 9.91 & 9.93 & 9.45 & 9.01 & 8.72 & 8.517 & 8.303 & 8.259 & 18307584 & $11,22,13,22,10,10,10,10,10,4,4,4$ \\
\hline
\end{tabular}

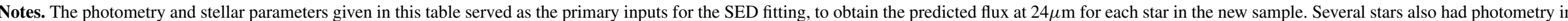

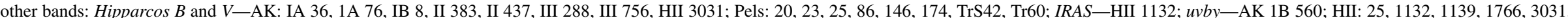
Tr60; Johnson JHK-HII 1766, 3031. The other bands were also included in the SED fitting, but are not listed in the table.

${ }^{\text {a }}$ Spectral type.

${ }^{\mathrm{b}}$ We estimated $\log g$ to be 4.5 when no data were available.

${ }^{c}$ We estimated $[\mathrm{Fe} / \mathrm{H}]$ to be 0 when no data were available.

${ }^{\mathrm{d}} T_{\text {eff }}$ was estimated using $B-V$ color where derived temperatures from spectra were not available. See references for sources of derived temperatures.

${ }^{\mathrm{e}} I_{C}$. were estimated from $V-I$ listed in the Hipparcos catalog. They are converted to $I_{J}$ using the following relation: $(V-I)_{C}=0.778(V-I)_{J}($ Bessell 1979$)$.

${ }^{\mathrm{f}}$ Pels 173: confusion of source. See the text for details.

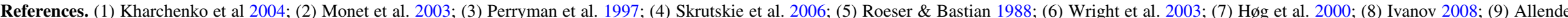

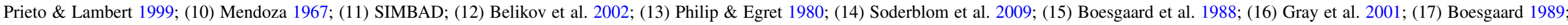

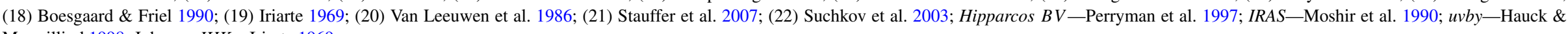
Mermilliod 1998; Johnson JHK-Iriarte 1969. 
Table 2

MIPS $24 \mu \mathrm{m}$ Fluxes and Derived Quantities for the Pleiades

\begin{tabular}{|c|c|c|c|c|c|c|c|c|c|}
\hline Target & $\begin{array}{c}F_{v} \text { (error) } \\
(\mathrm{mJy})\end{array}$ & Excess Ratio & $\Delta V$ & $\begin{array}{c}v \sin i \\
\left(\mathrm{~km} \mathrm{~s}^{-1}\right)\end{array}$ & $\begin{array}{c}\log L_{X}{ }^{\mathrm{a}} \\
\left(\log \left(\mathrm{erg} \mathrm{s}^{-1}\right)\right)\end{array}$ & $A_{*} / A_{\odot} \mathrm{b}$ & $L_{\mathrm{IR}} / L_{*}$ & Notes $^{c}$ & References \\
\hline AK IA 36 & $2.41(0.02)$ & 1.03 & 0.42 & 33.9 & - & 1.0 & $<4.9 \times 10^{-6}$ & PB & $1,4,-, 1$ \\
\hline AK 1 A 56 & $2.53(0.02)$ & 1.13 & -0.05 & 14.4 & - & 0.9 & $2.4 \times 10^{-5}$ & & $1,4,-,-$ \\
\hline AK 1A 76 & $4.59(0.05)$ & 1.26 & 0.01 & 11.7 & - & 1.6 & $2.5 \times 10^{-5}$ & & $1,4,-,-$ \\
\hline AK 1 A 317 & $3.17(0.04)$ & 1.01 & 0.00 & - & 30.0 & 1.4 & $<7.9 \times 10^{-7}$ & & $1,-, 7,-$ \\
\hline AK 1B 7 & $3.46(0.04)$ & 1.12 & 0.30 & - & - & 1.3 & $1.5 \times 10^{-5}$ & PB & $1,-,-, 1$ \\
\hline AK IB 8 & $2.13(0.02)$ & 1.04 & -0.10 & 11.9 & - & 1.1 & $<4.4 \times 10^{-6}$ & & $1,4,-,-$ \\
\hline AK 1B 146 & $3.62(0.06)$ & 0.93 & 0.16 & - & 28.3 & 1.2 & $<9.2 \times 10^{-6}$ & & $3,-, 7,-$ \\
\hline AK 1B 365 & $3.26(0.04)$ & 1.01 & 0.23 & 39.9 & - & 1.3 & $<7.2 \times 10^{-7}$ & PB & $1,4,-, 1$ \\
\hline AK 1B 560 & $4.37(0.04)$ & 1.34 & 0.11 & 21.6 & - & 1.5 & $3.2 \times 10^{-5}$ & & $1,4,-,-$ \\
\hline AK 1B 590 & $4.52(0.05)$ & 0.99 & -0.01 & - & - & 1.7 & $<5.9 \times 10^{-6}$ & & $1,-,-,-$ \\
\hline AK II 34 & $5.30(0.05)$ & 1.06 & 0.58 & 22.1 & 29.9 & 1.4 & $<1.1 \times 10^{-5}$ & PB & $1,4,7,12$ \\
\hline AK II 359 & $1.82(0.02)$ & 0.99 & 0.04 & 16.6 & - & 1.0 & $<6.5 \times 10^{-6}$ & & $1,4,-,-$ \\
\hline AK II 383 & $2.88(0.02)$ & 1.17 & 0.39 & 24.5 & 29.7 & 1.3 & $1.5 \times 10^{-5}$ & PB & $1,4,7,1$ \\
\hline AK II 437 & $9.89(0.09)$ & 3.79 & 0.18 & 29.6 & - & 1.4 & $2.4 \times 10^{-4}$ & & $1,4,-,-$ \\
\hline AK III 288 & $3.57(0.05)$ & 0.99 & 0.13 & 36.4 & - & 1.5 & $<6.3 \times 10^{-6}$ & & $1,4,-,-$ \\
\hline HII 25 & $4.13(0.05)$ & 1.12 & -0.02 & 46.0 & 29.1 & 1.6 & $1.1 \times 10^{-5}$ & & $1,4,7,-$ \\
\hline HII 102 & $2.72(0.02)$ & 1.04 & 0.44 & 24.58 & - & 0.7 & $<1.1 \times 10^{-5}$ & B; sep. $=3^{\prime \prime} .63$ & $1,5,-, 11$ \\
\hline HII 120 & 1.69 & 1.04 & -0.04 & 9.4 & 30.2 & 0.8 & $<6.3 \times 10^{-6}$ & & $2,4,9,-$ \\
\hline HII 152 & 1.82 & 1.12 & 0.02 & 11.3 & 30.6 & 1.0 & $1.3 \times 10^{-5}$ & & $2,4,9,-$ \\
\hline HII 173 & 2.10 & 1.00 & 0.61 & 5.2 & 28.9 & 0.6 & $<6.9 \times 10^{-7}$ & SB & $2,4,7,10,11$ \\
\hline HII 174 & 1.32 & 0.99 & -0.06 & 14.4 & 30.1 & 0.6 & $<1.5 \times 10^{-5}$ & & $2,4,7,-$ \\
\hline HII 250 & 1.90 & 1.12 & -0.06 & 7.1 & 29.4 & 0.9 & $1.4 \times 10^{-5}$ & & $2,4,7,-$ \\
\hline HII 293 & $1.80(0.05)$ & 0.98 & 0.01 & 6.6 & 29.2 & 0.8 & $<9.3 \times 10^{-6}$ & & $3,4,7,-$ \\
\hline HII 314 & 1.98 & 1.02 & -0.01 & 42.8 & 30.3 & 0.9 & $<2.4 \times 10^{-6}$ & & $2,4,7,-$ \\
\hline HII 405 & $2.94(0.08)$ & 0.98 & 0.10 & 18.5 & 29.7 & 1.4 & $<6.1 \times 10^{-6}$ & & $3,4,7,-$ \\
\hline HII 489 & $2.60(0.09)$ & 1.20 & -0.12 & 18.3 & 29.2 & 1.0 & $2.5 \times 10^{-5}$ & & $3,4,7,-$ \\
\hline HII 514 & 2.04 & 1.19 & 0.07 & 10.6 & 28.7 & 0.9 & $2.6 \times 10^{-5}$ & & $2,4,8,-$ \\
\hline HII 571 & $1.67(0.05)$ & 1.05 & -0.06 & 7.6 & - & 0.7 & $<1.1 \times 10^{-5}$ & & $3,4,-,-$ \\
\hline HII 727 & $3.68(0.08)$ & 1.00 & 0.29 & 66.4 & 29.8 & 1.2 & $<4.3 \times 10^{-7}$ & PB & $3,4,7,12$ \\
\hline HII 739 & $5.09(0.08)$ & 1.00 & 0.73 & 14.6 & 30.3 & 0.9 & $<7.8 \times 10^{-6}$ & PB & $3,4,7,12$ \\
\hline HII 923 & $2.77(0.08)$ & 1.06 & 0.09 & 18.3 & 29.2 & 1.1 & $<8.3 \times 10^{-6}$ & & $3,4,7,-$ \\
\hline HII 996 & $2.45(0.07)$ & 1.21 & -0.04 & 11.9 & 28.9 & 1.1 & $2.1 \times 10^{-5}$ & & $3,4,7,-$ \\
\hline HII 1015 & 1.77 & 0.98 & -0.03 & 9.6 & 29.3 & 1.1 & $<6.1 \times 10^{-6}$ & & $2,4,7,-$ \\
\hline HII 1101 & 3.30 & 1.48 & 0.00 & 20.0 & 29.6 & 1.1 & $5.3 \times 10^{-5}$ & & $2,4,7,-$ \\
\hline HII 1117 & $3.08(0.09)$ & 1.04 & 0.75 & 4.4 & 29.1 & 0.9 & $<9.8 \times 10^{-6}$ & SB & $3,4,7,10,11$ \\
\hline HII 1132 & $68.04(0.36)$ & 17.44 & 0.16 & 49.5 & - & 1.5 & $1.9 \times 10^{-3}$ & & $1,4,-,-$ \\
\hline HII 1139 & $4.03(0.06)$ & $1.09^{\mathrm{d}}$ & 0.08 & 31.8 & - & 1.8 & $<7.4 \times 10^{-6}$ & & $1,4,-,-$ \\
\hline HII 1182 & 1.93 & 1.03 & -0.03 & 16.6 & - & 1.0 & $<3.2 \times 10^{-6}$ & & $2,4,-,-$ \\
\hline HII 1200 & 3.09 & 1.15 & -0.07 & 13.7 & - & 1.3 & $1.6 \times 10^{-5}$ & & $2,4,-,-$ \\
\hline HII 1309 & $3.55(0.11)$ & 0.97 & -0.06 & 85.0 & 29.4 & 1.7 & $<5.2 \times 10^{-6}$ & & $3,6,7,-$ \\
\hline HII 1338 & $7.34(0.10)$ & 0.96 & 0.76 & 110.0 & 29.2 & 1.7 & $<1.0 \times 10^{-5}$ & $\mathrm{~B} ;$ sep. $=00^{\prime \prime} \cdot 2$ & $3,6,7,13$ \\
\hline HII 1514 & $2.11(0.07)$ & 1.07 & 0.06 & 13.7 & 29.2 & 1.0 & $<8.0 \times 10^{-6}$ & & 3,4,7,- \\
\hline HII 1613 & $2.83(0.08)$ & 1.01 & 0.01 & 20.0 & 29.3 & 1.4 & $<1.1 \times 10^{-6}$ & & $3,4,7,-$ \\
\hline HII 1726 & $5.25(0.10)$ & 1.04 & 0.66 & 12.9 & 29.5 & 1.4 & $<6.4 \times 10^{-6}$ & $\mathrm{VB} ;$ sep. $=0^{\prime \prime} .5$ & $3,4,7,10$ \\
\hline HII 1766 & $8.45(0.06)$ & 1.60 & 0.27 & 23.1 & - & 1.5 & $9.5 \times 10^{-5}$ & $\mathrm{~PB}$ & $1,4,-, 10$ \\
\hline HII 1797 & $3.68(0.08)$ & 1.52 & -0.13 & 19.8 & 29.4 & 1.4 & $4.3 \times 10^{-5}$ & & $3,4,7,-$ \\
\hline HII 1856 & $2.43(0.07)$ & 0.94 & -0.01 & 15.6 & 29.3 & 1.3 & $<5.3 \times 10^{-6}$ & & $3,4,7,-$ \\
\hline HII 1912 & $5.73(0.12)$ & 0.97 & 0.35 & 75.0 & 29.5 & 1.5 & $<1.1 \times 10^{-5}$ & $\mathrm{VB} ;$ sep. $=0^{\prime \prime} .3$ & $3,6,7,10$ \\
\hline HII 1924 & $2.24(0.07)$ & 1.04 & -0.08 & 14.2 & 29.2 & 1.2 & $<4.3 \times 10^{-6}$ & & $3,4,7,-$ \\
\hline HII 2027 & $2.24(0.06)$ & 0.97 & 0.63 & 2.4 & 29.6 & 0.6 & $<2.2 \times 10^{-5}$ & B; sep.:0.'10 & $3,4,7,11$ \\
\hline HII 2147 & 2.62 & 1.00 & 0.53 & 27.1 & 30.4 & 0.6 & $<1.6 \times 10^{-6}$ & SB & $2,4,7,11$ \\
\hline HII 2172 & $2.15(0.02)$ & $1.10^{\mathrm{d}}$ & -0.08 & - & 29.3 & 1.1 & $9.5 \times 10^{-6}$ & & $1,-, 7,-$ \\
\hline HII 2278 & 2.20 & 0.98 & 0.75 & 6.2 & 29.6 & 0.6 & $<2.2 \times 10^{-5}$ & B; sep. $=0 . ' 27$ & $2,4,7,11$ \\
\hline HII 2506 & 2.06 & 1.00 & -0.07 & 13.9 & 29.6 & 1.1 & $<3.4 \times 10^{-8}$ & & $2,4,9,-$ \\
\hline HII 2644 & 1.31 & 1.01 & 0.06 & 3.6 & 29.0 & 0.8 & $<7.4 \times 10^{-7}$ & & $2,4,7,-$ \\
\hline HII 2786 & 2.00 & 0.99 & -0.04 & 22.2 & 29.7 & 1.2 & $<5.3 \times 10^{-6}$ & & $2,4,9,-$ \\
\hline HII 2881 & 1.68 & 1.00 & 0.69 & 8.0 & 29.9 & 0.5 & $<1.6 \times 10^{-7}$ & B; sep. $=0.08$ & $2,4,7,11$ \\
\hline HII 3031 & $5.26(0.05)$ & $1.01^{\mathrm{d}}$ & 0.01 & - & 29.5 & 2.0 & $<1.2 \times 10^{-6}$ & & $1,-, 9,-$ \\
\hline HII 3097 & 1.65 & 1.01 & 0.06 & 14.7 & 29.8 & 0.8 & $<2.6 \times 10^{-6}$ & & $2,4,9,-$ \\
\hline HII 3179 & 2.41 & 1.00 & 0.00 & 5.0 & 29.6 & 1.2 & $<2.2 \times 10^{-7}$ & & $2,4,7,-$ \\
\hline PELS 7 & $2.16(0.02)$ & 0.99 & 0.74 & 2.7 & - & 1.1 & $<7.3 \times 10^{-8}$ & PB & $1,4,-, 1$ \\
\hline PELS 20 & $2.19(0.02)$ & 1.19 & -0.07 & 9.7 & - & 1.1 & $1.7 \times 10^{-5}$ & & $1,4,-,-$ \\
\hline PELS 23 & $2.90(0.04)$ & 1.06 & -0.05 & 36.2 & - & 1.1 & $<8.9 \times 10^{-6}$ & & $1,4,-,-$ \\
\hline PELS 25 & $3.33(0.04)$ & 1.02 & 0.15 & - & 29.8 & 1.6 & $<1.4 \times 10^{-6}$ & & $1,-, 7,-$ \\
\hline PELS 40 & $2.77(0.04)$ & 1.03 & 0.04 & 11.9 & - & 1.3 & $<2.8 \times 10^{-6}$ & & $1,4,-,-$ \\
\hline
\end{tabular}


Table 2

(Continued)

\begin{tabular}{|c|c|c|c|c|c|c|c|c|c|}
\hline Target & $\begin{array}{c}F_{v} \text { (error) } \\
(\mathrm{mJy})\end{array}$ & Excess Ratio & $\Delta V$ & $\begin{array}{c}v \sin i \\
\left(\mathrm{~km} \mathrm{~s}^{-1}\right)\end{array}$ & $\begin{array}{c}\log L_{X}{ }^{\mathrm{a}} \\
\left(\log \left(\mathrm{erg} \mathrm{s}^{-1}\right)\right)\end{array}$ & $A_{*} / A_{\odot} \mathrm{b}$ & $L_{\mathrm{IR}} / L_{*}$ & Notes $^{c}$ & References \\
\hline PELS 86 & $4.24(0.04)$ & 1.10 & 0.57 & - & - & 1.6 & $<9.2 \times 10^{-6}$ & PB & $1,-,-, 1$ \\
\hline PELS 121 & $2.45(0.02)$ & 0.97 & -0.11 & 4.9 & - & 1.0 & $<9.7 \times 10^{-6}$ & & $1,4,-,-$ \\
\hline PELS 124 & $3.01(0.04)$ & 1.06 & 0.19 & 20.4 & - & 1.4 & $<5.1 \times 10^{-6}$ & & $1,4,-,-$ \\
\hline PELS 128 & $2.90(0.10)^{f}$ & $1.05^{\mathrm{d}}$ & 0.70 & 4.8 & - & 1.0 & $<8.9 \times 10^{-6}$ & $\mathrm{~PB}$ & $1,4,-, 1$ \\
\hline PELS 135 & $4.35(0.04)$ & 1.10 & 0.05 & - & - & 1.5 & $<1.1 \times 10^{-5}$ & & $1,-,-,-$ \\
\hline PELS 146 & $3.00(0.03)$ & 1.43 & -0.10 & 17.8 & - & 1.0 & $6.3 \times 10^{-5}$ & & $1,4,-,-$ \\
\hline PELS 150 & $4.47(0.05)$ & 1.26 & 0.24 & 26.6 & - & 1.4 & $3.0 \times 10^{-5}$ & PB & $1,4,-, 1$ \\
\hline PELS 173 & $3.39(0.04)$ & $1.18^{\mathrm{d}}$ & -0.31 & 37.8 & - & 1.8 & $<1.1 \times 10^{-5}$ & & $1,4,-,-$ \\
\hline PELS 174 & $4.17(0.04)$ & 1.06 & 0.52 & 58.9 & 30.0 & 1.1 & $<1.1 \times 10^{-5}$ & $\mathrm{~PB}$ & $1,4,9,1$ \\
\hline $\operatorname{Tr} 60$ & $3.64(0.05)$ & 1.01 & 0.00 & - & 29.9 & 1.6 & $<7.5 \times 10^{-7}$ & & $1,-, 7,-$ \\
\hline $\operatorname{TrS} 42$ & $3.17(0.04)$ & 1.10 & 0.08 & 19.6 & - & 1.4 & $1.0 \times 10^{-5}$ & & $1,4,-,-$ \\
\hline
\end{tabular}

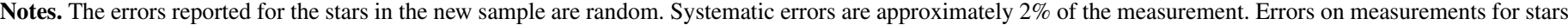
taken from Stauffer et al. (2005) and Gorlova et al. (2006) are estimated to be about the same.

${ }^{a}$ Upper limits on $\log L_{x}$ were not computed due to discrepancies between sources of X-ray photometry.

b Where $A_{\odot}=6.1 \times 10^{22} \mathrm{~cm}^{2}$.

${ }^{c}$ B: binary; PB: photometry binary; SB: spectroscopic binary.

${ }^{\mathrm{d}}$ Excess ratios not included in Figure 1. See the text for details.

e Aperture photometry.

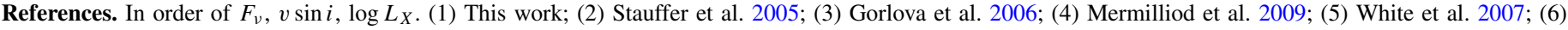

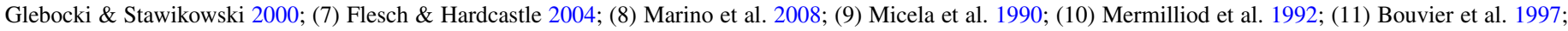
(12) Kähler 1999; (13) Mason et al. 2003.

\subsubsection{Photometric Colors}

Extrapolation of the photospheric SED using photometric colors, e.g., $V-K_{S}$ versus $K_{S}-$ [24], is a relatively easy method to apply to large samples of stars. It has the convenient feature that it is self-calibrating. Since many stars can be anticipated to have no excess emission at $24 \mu \mathrm{m}$, there is a strong peak at small values of the excess ratio that can be set to one. There is a risk that small excesses will be lost through this approach. In comparison, SED fitting as in Section 3.1.1 has the advantage that it retains the absolute SED level, but with the disadvantage that the level deduced depends on the accuracy of the theoretical photosphere models. In this paper, we will place color-based extrapolation on an absolute scale and then compare the results with those from SED fitting to probe these advantages and disadvantages.

We used the FEPS sample (Carpenter et al. 2008) to probe different approaches to using photometric colors, after checking that the $24 \mu \mathrm{m}$ photometry in that paper is consistent with ours. For this sample, we can use 2 MASS $J H K_{S}$ photometry to anchor photospheric predictions, since almost all the members are fainter than the 2MASS saturation limit. The FEPS team also obtained IRAC photometry at three bands for the sample. To select a subsample that should have very few stars with excess emission, we used chromospheric activity as an indicator of stellar age and accepted only those stars indicated to be older than 500 Myr in the calibration of Mamajek \& Hillenbrand (2008). We used the width of the peak in excess ratio, $W_{\text {ex }}$, as an indicator of the quality of the extrapolation; this metric is not influenced by a small number of outliers (e.g., stars with excess emission).

Carpenter et al. (2008) derive a number of small corrections to the IRAC and MIPS photometry based on the behavior of the sample as a whole. We found that application of these corrections either in the IRAC bands or at $24 \mu \mathrm{m}$ increased $W_{\text {ex }}$. The best performance was obtained by using just the uncorrected $8 \mu \mathrm{m}$ photometry, yielding an rms scatter of $1.6 \%$ in the extrapolated flux density at $24 \mu \mathrm{m}$ compared with the measured values.
For many stars listed in Table 2, including the new sample of Pleiades stars, IRAC photometry is not available; 2MASS (or other $J H K$ ) photometry must be used for SED extrapolations. We found that this extrapolation was most accurate when the $K_{S^{-}}$ band measurement was combined with the $H$-band measurement using the nominal $H-K$ color for the spectral type of the star and weighting by the quoted $2 \mathrm{MASS}$ errors. To do this, we plotted $V-K_{S}$ versus $H-K_{S}$ and used a fit to determine $V-K_{S}$, which we used to estimate the stellar types. Addition of the $J$-band data decreased the accuracy, presumably because the $J-K_{S}$ color is a relatively strong function of spectral type and the derived spectral types have significant errors. The result using just the $H$ - and $K_{S}$-band data provided rms deviations of $2.5 \%$ in the extrapolated flux density at $24 \mu \mathrm{m}$, i.e., about 1.5 times the scatter obtained with the $8 \mu \mathrm{m}$ band. This study allowed us to set the value of 89 for the ratio of $K_{S}$ band to $24 \mu \mathrm{m}$ flux densities that gives an excess ratio of one for stars with no excess. This value agrees to within $1.4 \%$ with the similar value from the calibration of Rieke et al. (2008), that is, within the quoted error there of $2 \%$. We use the value derived from the FEPS data to analyze our observations of the new sample.

\subsubsection{Final Excess Ratios}

For the new sample, the excess ratios determined by SED fitting and by photometric colors are of comparable quality. They are generally in agreement with an rms difference of $3 \%$. We therefore averaged the two determinations. The final value of the rms scatter for the stars with no evidence for excesses is $3.6 \%$. Given typical errors for the 2MASS $K_{S}$ magnitudes (even after combining with the $H$-band magnitudes of $2 \%$ and the expected noise level of the observations $(\sim 3 \%))$, this value is reasonable. It is only slightly worse than the noise level achieved on much brighter field stars (e.g., Su et al. 2006; Trilling et al. 2008), showing that no additional error sources intrude at the much lower signal levels of the Pleiades stars.

Stauffer et al. (2005) used IRAC $8 \mu \mathrm{m}$ photometry for their SED extrapolations. We put their results on the same scale as 


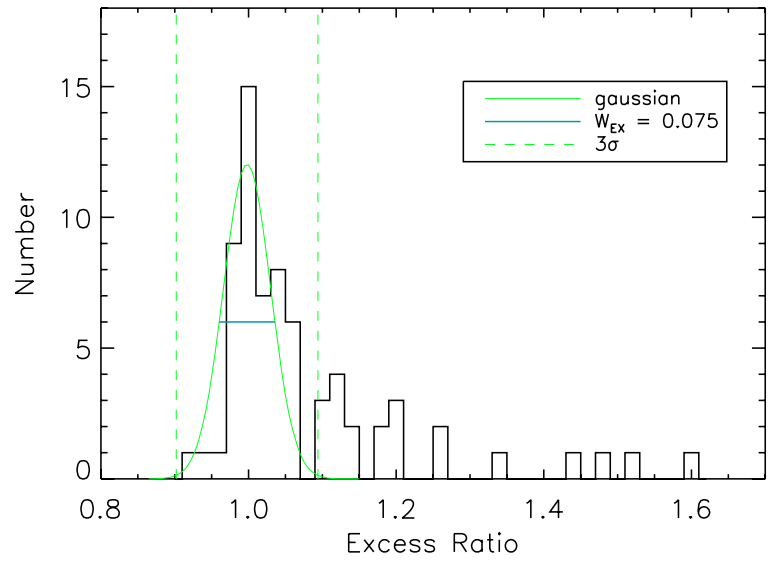

Figure 1. Distribution of $24 \mu \mathrm{m}$ excess ratios for Pleiades members in Table 2. A Gaussian has been fitted to show the photospheric behavior along with the effect of the errors in extrapolating it to $24 \mu \mathrm{m}$, with the width of the peak, $W_{\mathrm{EX}}$, indicated in the plot. The stars with values above and to the right of the Gaussian show infrared excess emission. Two stars with extreme excesses, AK II 437 and HII 1132, are not included in the figure.

(A color version of this figure is available in the online journal.)

those for the new sample by applying the corrections derived in Rieke et al. (2008). We augmented the results of Gorlova et al. (2006) with 2MASS $H$-band photometry converted to the $K_{S}$ band as described above, in all cases where the indicated $K_{S}$-band error was greater than 0.02 . Table 2 includes the $24 \mu \mathrm{m}$ fluxes and excess ratios for the full sample.

The distribution of excess ratios for the combined sample is plotted in Figure 1, which also shows a Gaussian fit to the distribution. The fit was optimized by computing chi-squared over a window centered at the derived fit center and extending to the point at $\sim 15 \%$ of the distribution peak on either side. This approach was designed to reduce the effect on the fitted parameters by stars with excesses. The final fit is centered at 0.998 with a standard deviation of 0.032 .

\section{RESULTS}

From Figure 1, excess ratios $>1.1$ are identified as having infrared excesses at $24 \mu \mathrm{m}$. This corresponds to $3 \sigma$ of the Gaussian fit shown. The incidence of excesses for ratios $>1.06$ can be estimated by subtracting the fit to the no-excess distribution from the observed numbers of stars with excesses in this range. Out of a total combined sample membership of 71 stars, 23 have excess ratios exceeding the $3 \sigma$ level. Fourteen have ratios $>1.16$ (or $>5 \sigma$ ) for comparison with the study of Praesepe by Gáspár et al. (2009). An additional six stars may have excess ratios between 1.06 and 1.10. By definition, subtracting these numbers from the observed distribution leaves the values fitted adequately by the Gaussian for no excess. Since its center is not significantly offset from one, it is likely that these remaining 42 stars have very little excess emission down to a low level. Expressed as percentages, $20 \% \pm 5.3 \%$ have excess ratios $>5 \sigma$, $32.4 \% \pm 6.8 \%$ have them $>3 \sigma$, and about $41 \% \pm 7.6 \%$ have them $>1.06(\sim 1.7 \sigma)$. These values are higher then previous estimates for the incidence of excess in the Pleiades, but consistent with the old ones within the errors and given the identification of excess at smaller flux levels in this analysis. The remaining $59 \%$ have no excess exceeding the $1.7 \sigma$ level.

Because we include in our analysis stars from previous studies, we will address some possible biases in our results. We include HII 1338 and 1912, which do not meet the color- selection criteria. Since early-type stars in general may have a higher incidence rate of $24 \mu \mathrm{m}$ excesses than later-type ones, a bias toward stars with excess emission might result. However, neither of these stars has an excess, adding no bias to the sample in that regard. We also rejected other stars due to low signal-tonoise ratio (see Section 2). Rejecting stars with low signal-tonoise ratio might bias the sample in favor of excesses; however, only one of these stars (HII 1095) has any indication of an excess (in this case at the $4 \sigma$ level of significance), so the incidence of excesses among these stars is approximately the same as we derive for the full sample and no significant bias is likely. Therefore, we are confident that the excess rates derived above are correct for our sample.

\section{DISCUSSION}

Our study of the Pleiades probes the debris disk characteristics in a large sample of solar-type stars of well-determined age. We compare our sample to similar studies done with A stars and the Blanco 1 open cluster. We show that debris disks emitting at $24 \mu \mathrm{m}$ are still common at $100 \mathrm{Myr}$ around both A- and solartype stars, which provide an important constraint on theoretical models for their dissipation and replenishment. We also use this sample to examine possible correlations with other stellar parameters. Normally, the dramatic decay of infrared excesses with age, combined with the uncertainties in stellar ages, can mask important second-parameter effects. However, since age variation is removed in the studies of the Pleiades and other open clusters, they provide the best opportunity to examine other influences on debris disk behavior.

\subsection{Comparison of Incidence of Excesses}

Our value for the incidence of excesses in the Pleiades can be compared with that of Stauffer et al. (2009) on the Blanco 1 cluster. This cluster is similar in age to the Pleiades $(\sim 100 \mathrm{Myr})$ but about twice as far away $(\sim 250 \mathrm{pc})$. Within the spectral type range for our Pleiades study $\left(1.05 \leqslant V-K_{S} \leqslant 2.15\right)$, there are 18 Blanco 1 members observed at $24 \mu \mathrm{m}$. Two of them, W91 and W99, are identified as having $24 \mu \mathrm{m}$ excesses at $>3 \sigma$ (Stauffer et al. 2009). Three more, W38, W53, and Z5102, have excesses indicated at $>2.5 \sigma$. The method used to estimate $\sigma$ in this paper, comparing photometry of sources observed in more than one pointing, is very conservative as applied to sources targeted for photometry. The latter objects are centered in the field and measured in exactly the way used for calibration stars, on which the standard sensitivity estimates are based. The former objects tend to lie near field edges and would be expected to have lower-accuracy measurements. In fact, the Spitzer Science Center sensitivity estimator suggests that the $1 \sigma$ level for the targeted objects in the Stauffer et al. (2009) survey should be about $25 \mu \mathrm{Jy}([24]=10)$, which is nearly twice as accurate as indicated in Stauffer et al. (2009). We therefore consider W38, W53, and Z5102 to have reliably measured excesses also. We adopt a net count of 5 excesses among 18 stars, or an incidence of $\sim 28 \%$. This result is close to the one for the Pleiades, albeit at much lower statistical significance.

We can also compare our Pleiades sample with the members of the sample of A stars selected from Su et al. (2006) whose ages are between 50 and 300 Myr. This study has a "zeropoint"excess ratio for no excess of 0.981 and $1 \sigma=0.026$, compared with 0.998 and 0.032 , respectively, for our Pleiades sample. Therefore, to compare at similar detection thresholds, we determine the number of A stars with excess ratios above 


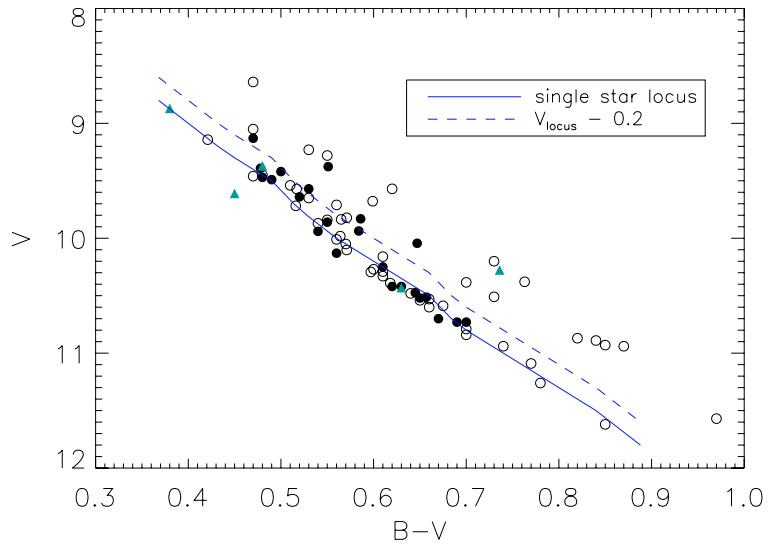

Figure 2. $V$ vs. $B-V$ for the Pleiades combined sample, listed in Table 2. The triangles represent HII 1139, HII 2172, HII 3031, Pels 128, and Pels 173, which were not included in the final analysis (see the text). The circles represent the rest of the stars, with the filled ones having $24 \mu \mathrm{m}$ excesses $>3 \sigma$. The lines represent the main-sequence locus for the Pleiades (solid) and the cutoff for binarity (dashed).

(A color version of this figure is available in the online journal.)

1.08 and 1.13 for $3 \sigma$ and $5 \sigma$, respectively. Of 69 A stars within this age range, 21 have excess $>5 \sigma$ and 25 have them $>3 \sigma$, corresponding to $30 \% \pm 6.6 \%$ and $36 \% \pm 7.2 \%$, respectively. The latter value is slightly higher than the corresponding one for solar-type stars, i.e. (combining the Pleiades and Blanco 1), 28 of 89 , or $31 \% \pm 5.9 \%$, but the difference is within the statistical uncertainties.

The relative weakness of this dependence on spectral type at the age of the Pleiades can be contrasted with the virtual complete absence of $24 \mu \mathrm{m}$ excesses around solar-type stars at 750 Myr (Gáspár et al. 2009). It appears that old A-type stars have a higher incidence of excesses, although they too experience a rapid decline in this regard after $\sim 100$ Myr (Gáspár et al. 2009). There may be a relatively simple explanation for this type of difference in excess evolution. The A-type stars have typically 2.5 times the mass and 25 times the luminosity of the solar-type ones. The thermal equilibrium distance from the star for grains emitting at $24 \mu \mathrm{m}$ will be five times greater for the A stars, and the Keplerian orbital velocities of the grains will be square root of two less. Thus, the collisional evolution timescales for the portions of the debris systems that dominate the $24 \mu \mathrm{m}$ emission should be longer for the A stars than for the solar-type ones. These scalings also show that all the dust production and clearing timescales will be longer for the A-type stars (Dominik \& Decin 2003), again consistent with the slower decay of the excesses around them. We can also relate this to our own Sun. Combining our excess rate at $100 \mathrm{Myr}$ with that at $750 \mathrm{Myr}$, we estimate that only a small fraction of solar-type stars at the age of the Sun, if any, will have detectable excesses at $24 \mu \mathrm{m}$, assuming a steady decay rate. An analysis of the excess decay timescales as a detailed function of stellar mass, e.g., using the full Spitzer data set, may be able to test this basic hypothesis and to refine it into a test of models for debris disk evolution.

Carpenter et al. (2009a) present a similar study, comparing the excess fractions of early- and solar-type stars at various ages by compiling data from several previous works (see Figure 6 and Table 4 in their paper). From Figure 6 in their paper, at $100 \mathrm{Myr}$, the excess fractions between early- and solar-type stars differ significantly, with the latter type having the lower excess fraction. However, Carpenter et al. define solar-type stars as being from $\mathrm{G} 0$ to $\mathrm{K} 5$ in spectral type, whereas we have defined

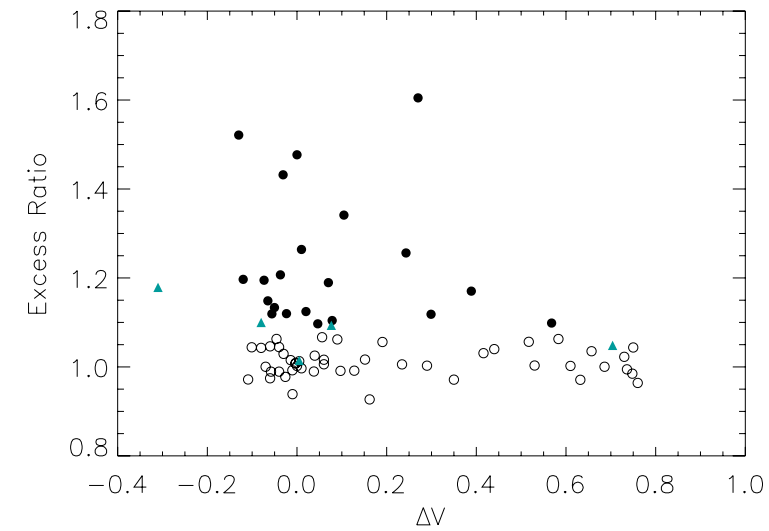

Figure 3. Excess ratio vs. $\Delta V$ for the Pleiades combined sample, where $\Delta V$ is the difference between the observed $V$ magnitude and the main sequence. There is an anti-correlation between $24 \mu \mathrm{m}$ excess and binarity. The triangles represent the five stars removed from analysis (HII 1139, HII 2172, HII 3031, Pels 128, and Pels 173). AK II 437 and HII 1132 are not shown.

(A color version of this figure is available in the online journal.)

solar type as F5 to K1, placing a G2 star more in the center of the range, in regard to mass. Using color as an approximate indication of spectral type, we count 27 stars in our full sample as $\mathrm{F}$ type, and the remaining 44 as $\mathrm{G}$ or $\mathrm{K}$ type. Of the $27 \mathrm{~F}$ type stars, 12 have excesses giving a fraction of $44 \%$. Of the G- and K-type stars, 11 have excesses giving a fraction of $25 \%$. We attribute the difference in results between our paper and Carpenter et al. (2009a) as due to the difference in definition of solar-type stars.

\subsection{Excesses and Binarity}

Stauffer et al. (2009) found that binary stars in Blanco 1 and NGC 2547 tended not to have $24 \mu \mathrm{m}$ excesses. Gorlova et al. (2006) previously found similar results for stars in the Pleiades. We use similar methods to probe this correlation for our combined sample. Figure 2 shows $V$ versus $B-V$ for the stars listed in Table 2 . Possible binary systems were found fitting the photometry to the single star locus found in Stauffer et al. (2007). The difference between the observed $V$ magnitude and the single-star locus, or $\Delta V$, for each star is listed in Table 2; stars with $\Delta V$ of greater than 0.2 are likely to be binaries. To test whether binarity affects the excess rate for our sample, in Figure 3 we show excess ratio versus $\Delta V$. The trend is very similar to that reported by Stauffer et al. (2009) and confirms the anti-correlation between binarity and $24 \mu \mathrm{m}$ excess. Both stars with extreme excesses, AK II 437 and HII 1132, follow this trend, although they are not shown in Figure 3. To test this trend for the higher-mass binary systems, we used a KolmogorovSmirnov $(\mathrm{K}-\mathrm{S})$ test, which revealed an $8 \%$ probability that there is no anti-correlation between excess and high-mass binarity, assuming a cutoff of 0.4 for $\Delta \mathrm{V}$.

Most of the Pels and AK stars have not been well studied for binarity. However, separations for stars previously identified as binaries are listed in Table 2. The typical separation is less than 0.5 , which corresponds to $\sim 70 \mathrm{AU}$ at the distance of the Pleiades. Given the separations, it is plausible that the binary companions are disrupting any possible disks around these stars. In fact, Figure 4 of Trilling et al. (2007) shows a general absence of $24 \mu \mathrm{m}$ emission for field binaries with similar separations to those that are typical in the Pleiades, and they explain the effect as being a result of the disruptive effect of a binary companion at a distance from the star where a debris disk could typically be located. 


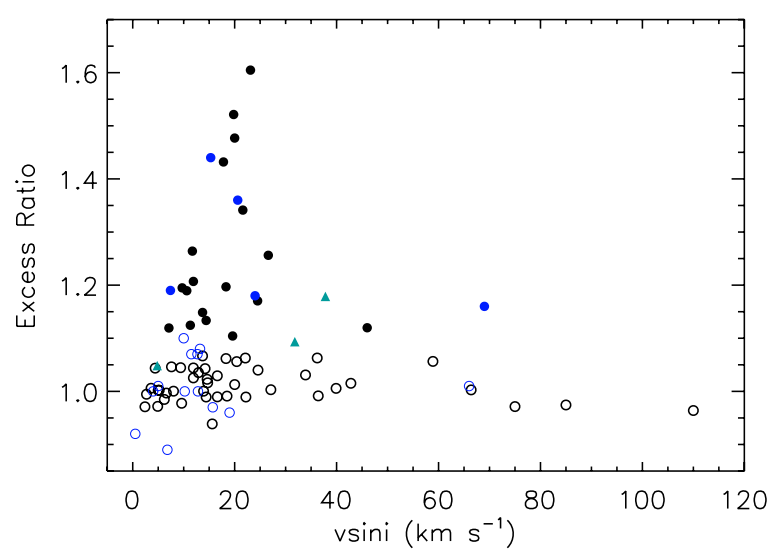

Figure 4. Excess ratio vs. $v \sin i$ for the Pleiades and Blanco 1. Pleiades stars are in black; Blanco 1 stars in blue. Stars removed from the Pleiades sample (HII 1139, Pels 128, and Pels 173) are represented with triangles. AK II 437 and HII 1132 are not shown.

(A color version of this figure is available in the online journal.)

\subsection{Correlations of Excesses with $X$-ray Activity and Rotation}

In general, for solar-type stars, rotation rate correlates with Xray luminosity (Noyes et al. 1984); both correlate inversely with the age and may correlate directly with the strength of the stellar wind (Wood et al. 2005; Holzwarth \& Jardine 2007; Cranmer 2008). There are a variety of reasons to expect correlations among these parameters and the incidence of infrared excesses. For example, Currie et al. (2008) find an inverse correlation between the stellar rotation rate and the amount of material available to produce a debris disk among early-type stars in the young cluster NGC 2232, and suggest that massive protostellar disks allow stars to spin down efficiently so that an inverse correlation is to be expected. A possible issue with applying this argument to excesses at $24 \mu \mathrm{m}$ is that most of the mass in a debris disk is typically in the cold component that dominates the $70 \mu \mathrm{m}$ emission, so the correlation may not be apparent at the shorter wavelength. Another possibility is that strong stellar winds remove debris dust quickly (Plavchan et al. 2005; Minato et al. 2006). The difficulty in confirming this prediction lies in the uncertainties in determining stellar winds, as is made clear in the review by Cranmer (2008). The number of stars with direct wind measurements is small; they indicate a roughly linear dependence on X-ray emission but with a substantial deviation downward at high X-ray luminosity (Wood et al. 2005).

By removing the age dependence of the infrared excesses, observations in clusters let us probe for any relations with these other parameters. Below, we consider rotation and X-ray surface brightness (and presumably stellar winds).

\subsubsection{Rotation}

We use the measured $v \sin i$ as an indicator for stellar rotation rate. In Figure 4, we show the excess ratio at $24 \mu \mathrm{m}$ versus $v \sin i$ for the stars in Table 2. Each star with a significant excess has $v \sin i<50 \mathrm{~km} \mathrm{~s}^{-1}$, including both stars with extreme excesses (AK II 437 and HII 1132). Stauffer et al. (2009) found a similar effect in the Blanco 1 data. Rebull et al. (2008) also found a slightly inverse trend between excess and rotation for $\mathrm{G}, \mathrm{K}$, and $\mathrm{M}$ stars in the $\beta$ Pic moving group, but suggested as an alternative explanation that it could be due to stellar wind.

However, in all these cases, the number of stars in the samples also decreases with increasing $v \sin i$, making it necessary to test for selection bias. To do so, we have combined the

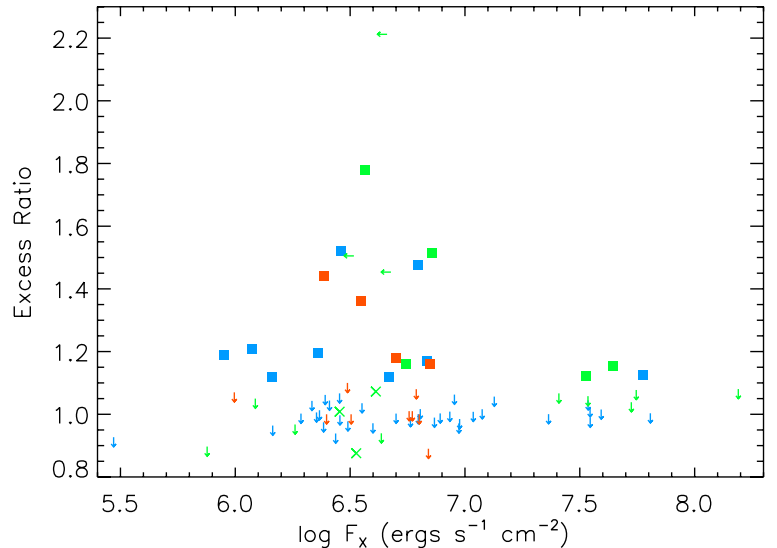

Figure 5. Excess ratio vs. $\log X$-ray surface brightness $\left(\log F_{X}\right)$ for the Pleiades, NGC 2547, and Blanco 1 clusters. Stars with excesses are represented by filledin squares. Upper limits on $F_{X}$ are represented by left-pointing arrows; upper limits on excess ratios by down-pointing arrows. Upper limits on both quantities are shown with cross signs. Pleiades stars are shown in blue, NGC 2547 in green, and Blanco 1 in red.

(A color version of this figure is available in the online journal.)

Pleiades and Blanco 1 samples (after removing from the former stars with extreme excesses that probably arise through recent large events (HII 1132 and AK II 437)). A K-S test gives a $38 \%$ probability that the correlation arises by chance, using $50 \mathrm{~km} \mathrm{~s}^{-1}$ as the boundary between slow and fast rotation. After the eliminations listed above, there are only 7 stars with $v \sin i$ above that boundary, compared to 72 stars below. With a cutoff of $30 \mathrm{~km} \mathrm{~s}^{-1}$, the probability increases to $49 \%$. Figure 4 is suggestive of a correlation between $24 \mu \mathrm{m}$ excess and rotation because none of the fast rotators have excesses; however, because there are so few stars with large $v \sin i$, the apparent correlation could be the result of small number statistics.

Stellar rotation has also been studied in a number of young clusters through high-accuracy photometry (Aigrain et al. 2007). However, the overlap between the stars with measured rotation periods and those studied for excesses with Spitzer is inadequate to extend this test. Within the limitations of current data, we conclude that there is no evidence for an inverse correlation of infrared excess at $24 \mu \mathrm{m}$ with rotation, at least for solar-type stars of $\sim 100 \mathrm{Myr}$ age.

\subsubsection{Stellar Winds}

The Pleiades stars generally show an inverse relationship between X-ray luminosity and the presence of $24 \mu \mathrm{m}$ excesses. Figure 5 shows the excess ratio at $24 \mu \mathrm{m}$ versus X-ray surface brightness for members of the Pleiades, NGC 2547, and Blanco 1 , all of which seem to show this effect. Similar behavior was found previously for much younger stars in the ScorpiusCentaurus OB association (Chen et al. 2005).

Such behavior might be associated with stellar winds. There are several mechanisms for clearing dust grains in debris disks, including particle-particle collisions and, when the grains are small enough, Poynting-Robertson drag, stellar wind drag, and photon pressure (Plavchan et al. 2009; Gustafson 1994, and references therein). The relative roles of these grainremoval mechanisms depend on the age and spectral type of the star (Plavchan et al. 2009, 2005). Dominik \& Decin (2003) and Wyatt (2005) show that grain-grain collisions are more important than Poynting-Robertson drag for grain removal in typical mature debris disks. However, winds act in a similar way to Poynting-Robertson drag to cause grains to spiral 
Table 3

Fit Parameters for Isochrones

\begin{tabular}{lrccc}
\hline \hline Cluster & Age & {$[\mathrm{Fe} / \mathrm{H}]$} & $A_{V}$ & References \\
\hline Pleiades & 115 & +0.03 & 0.12 & $1,2,3$ \\
NGC 2547 & 35 & -0.26 & 0.19 & $4,5,5$ \\
Blanco 1 & 90 & +0.04 & 0.06 & $6,7,8$ \\
\hline
\end{tabular}

References. (1) Meynet et al. 1993; Stauffer et al. 1998; Martín et al. 2001; (2) Soderblom et al. 2009; (3) Stauffer \& Hartmann 1987; (4) Jeffries \& Oliveira 2005; (5) Claria 1982; (6) Panagi \& O’Dell 1997; (7) Ford et al. 2005; (8) Westerlund et al. 1988.

into the star. The strong winds typical in young stars can become the dominant grain-removal mechanism for winds greater than three times the solar value, so long as the amount of dust in the relevant part of the debris system is moderate (Minato et al. 2006; Plavchan et al. 2009). Plavchan et al. (2009) show that stellar winds affect the evolution of debris disks around $\mathrm{K}$ and $\mathrm{M}$ dwarfs, and suggest that they could also be important for young solar-type stars.

We have investigated this possibility by computing approximate X-ray surface brightnesses, $F_{x}$ (which should be roughly correlated with wind strength), and comparing these values with excess ratio. We obtained stellar surface areas $\left(A_{*}\right)$ by estimating stellar luminosities, masses, and temperatures from an isochrone (Marigo et al. 2008; Bonatto et al. 2004) fitted to the stars' $M_{K}$ and $V-K_{S}$ measurements. Fitting parameters for the isochrones for each cluster are listed in Table 3. In keeping with our sample selection for the Pleiades, we selected from the NGC 2547 and Blanco 1 subsamples stars with $1.05<V-K_{S}<2.15$ to limit our analysis to solar-type stars.

We computed the fractional infrared luminosities as $\sim v L_{v}(24 \mu \mathrm{m})$, assuming the disk temperatures are about $150 \mathrm{~K}$. This temperature yields a lower limit to the true fractional luminosity. This assumes that the stars are single stars, but we have already shown that another removal mechanism tends to dominate for binaries. Upper limits on the fractional luminosities for those stars with excess ratios less than 1.0 were assumed to have a maximum excess ratio of 1.064 , corresponding to $2 \sigma$ above the photosphere. We assumed cluster distances of $407 \mathrm{pc}$ and 250 pc for NGC 2547 and Blanco 1, respectively (Mayne \& Naylor 2008; Panagi \& O’Dell 1997). For the Pleiades, we assumed the more traditional distance of $130 \mathrm{pc}$.

We used the X-ray surface brightnesses to estimate the stellar wind strength. There are indications that any relation between wind and X-ray properties may saturate at large values (Wood et al. 2005; Cranmer 2008), producing significant differences between the observed and predicted wind strengths. But because the saturation level is not well determined, we use the formula proposed by Wood et al. (2005) scaled to 36 Oph to estimate the dependence of the stellar wind strength on the X-ray surface brightness. Below, we discuss each cluster individually.

Pleiades. We used ROSAT X-ray fluxes. For HII 1797, we fitted the isochrone to its $B-V$ color instead of $V-K_{S}$, as 2MASS $K_{S}$ was not available. Pleiades X-ray luminosities and fractional infrared luminosities are listed in Table 2. Only one star, HII 152, has a significant excess and a strong estimated stellar wind. Neither star with an extreme excess, AK II 437 and HII 1132, has ROSAT data.

$N G C$ 2547. For those NGC 2547 members with $24 \mu \mathrm{m}$ detections, X-ray luminosities and $V$ magnitudes were obtained from Jeffries et al. (2006). Gorlova et al. (2007) identified stars with excesses on a $V-K$ versus $K-$ [24] plot. We found an approximate excess ratio for each star and set 1.10 as the threshold for having a meaningful $24 \mu \mathrm{m}$ excess for NGC 2547, similar to that for the Pleiades. The excess ratios and fractional luminosities for NGC 2547 members are listed in Table 4 by right ascension (R.A.) and declination (decl.). Two stars in NGC 2547 (R.A., decl.: 122.446875, -49.21808333 and 122.5642083, -49.09686111) show strong winds and excesses. There are eight stars in total that meet our $V-K$ color criteria and are reasonably likely to have $24 \mu \mathrm{m}$ excesses (Gorlova et al. 2007). Assuming upper limits of $\log L_{X}<29.3$ for the ones not detected, then six stars with infrared excess have weak winds. This is a conservative estimate of the upper limit on $\log L_{X}$ given that nearly half of the stars in the sample were detected having

Table 4

MIPS $24 \mu \mathrm{m}$ Magnitudes, Excess Ratios, Mass Loss Rates, and Fractional Luminosities for NGC 2547

\begin{tabular}{|c|c|c|c|c|c|c|c|}
\hline R.A. & Decl. & $\begin{array}{c}{[24]} \\
(\mathrm{mag})\end{array}$ & $\begin{array}{c}V-K_{S} \\
(\mathrm{mag})\end{array}$ & Excess Ratio & $\begin{array}{c}\log L_{X} \\
\left(\log \left(\operatorname{erg~s}^{-1}\right)\right)\end{array}$ & $A_{*} / A_{\odot}$ & $L_{\mathrm{IR}} / L_{*}$ \\
\hline 122.345375 & -49.13269444 & 10.26 & 1.31 & 1.78 & 29.4 & 1.1 & $8.9 \times 10^{-5}$ \\
\hline 122.446875 & -49.21808333 & 10.67 & 1.48 & 1.15 & 30.4 & 0.9 & $2.7 \times 10^{-5}$ \\
\hline 122.47525 & -49.32247222 & 10.97 & 1.79 & 0.94 & 29.3 & 0.7 & $<1.7 \times 10^{-5}$ \\
\hline 122.5161667 & -49.01861111 & 10.85 & 1.65 & 1.04 & 30.4 & 0.8 & $<9.2 \times 10^{-6}$ \\
\hline 122.538704 & -49.348375 & 10.74 & 1.64 & 1.07 & $<29.3$ & 0.8 & $<1.8 \times 10^{-5}$ \\
\hline 122.539 & -49.01541667 & 10.35 & 2.06 & 1.08 & 30.7 & 0.6 & $<6.2 \times 10^{-5}$ \\
\hline 122.5485417 & -49.37413889 & 10.22 & 1.41 & 0.97 & 29.1 & 1.0 & $<6.2 \times 10^{-5}$ \\
\hline 122.556 & -49.34547222 & 9.65 & 1.26 & 1.05 & 29.0 & 1.2 & $<1.5 \times 10^{-5}$ \\
\hline 122.5642083 & -49.09686111 & 10.54 & 1.88 & 1.12 & 30.1 & 0.6 & $5.5 \times 10^{-5}$ \\
\hline 122.576542 & -49.112867 & 10.01 & 1.43 & 1.51 & $<29.3$ & 1.0 & $1.1 \times 10^{-4}$ \\
\hline 122.605208 & -49.316325 & 10.72 & 1.45 & 0.88 & $<29.3$ & 1.0 & $<1.1 \times 10^{-5}$ \\
\hline 122.612625 & -49.14708333 & 11.29 & 1.84 & 1.06 & 30.1 & 0.7 & $<1.3 \times 10^{-5}$ \\
\hline 122.615 & -49.20263889 & 9.81 & 1.31 & 0.90 & 28.7 & 1.1 & $<1.8 \times 10^{-5}$ \\
\hline 122.6446667 & -49.14447222 & 10.85 & 1.47 & 1.07 & 30.2 & 1.0 & $<1.1 \times 10^{-5}$ \\
\hline 122.665958 & -49.077206 & 10.29 & 1.30 & 1.01 & $<29.3$ & 1.2 & $<1.6 \times 10^{-6}$ \\
\hline 122.6906667 & -49.01888889 & 10.28 & 1.40 & 1.51 & 29.7 & 1.0 & $8.3 \times 10^{-5}$ \\
\hline 122.7079167 & -49.19052778 & 10.28 & 1.33 & 1.16 & 29.7 & 1.4 & $1.9 \times 10^{-5}$ \\
\hline 122.750438 & -49.112367 & 10.65 & 1.76 & 2.21 & $<29.3$ & 0.7 & $2.0 \times 10^{-4}$ \\
\hline 122.758417 & -49.269381 & 10.90 & 1.81 & 1.45 & $<29.3$ & 0.7 & $1.0 \times 10^{-4}$ \\
\hline 122.7596667 & -49.27327778 & 10.47 & 1.81 & 1.08 & 30.4 & 0.7 & $<3.4 \times 10^{-5}$ \\
\hline
\end{tabular}


Table 5

Derived Quantities for Blanco 1

\begin{tabular}{lccr}
\hline \hline Name & $A_{*} / A_{\odot}$ & $\log L_{X}$ & $L_{\mathrm{IR}} / L_{*}$ \\
\hline W 38 & 1.6 & 29.85 & $1.5 \times 10^{-5}$ \\
W 53 & 1.2 & 29.58 & $2.0 \times 10^{-5}$ \\
W 56 & 1.3 & 29.4 & $<1.1 \times 10^{-7}$ \\
W 58 & 1.5 & 29.74 & $<1.2 \times 10^{-6}$ \\
W 68 & 1.5 & 28.95 & $<6.4 \times 10^{-6}$ \\
W 70 & 1.2 & $\ldots$ & $<8.3 \times 10^{-6}$ \\
W 79 & 1.0 & $\ldots$ & $<9.8 \times 10^{-6}$ \\
W 89 & 1.1 & 29.38 & $<7.7 \times 10^{-6}$ \\
W 91 & 1.1 & 29.3 & $4.5 \times 10^{-5}$ \\
W 99 & 1.4 & 29.31 & $<.3 \times 10^{-5}$ \\
W 113 & 1.1 & 29.4 & $<2.1 \times 10^{-5}$ \\
ZS 62 & 0.7 & $\ldots$ & $<1.9 \times 10^{-5}$ \\
ZS 83 & 0.6 & $\ldots$ & $<3.8 \times 10^{-7}$ \\
ZS 95 & 0.6 & $<29.43$ & $2.7 \times 10^{-5}$ \\
ZS 100 & 0.6 & $\ldots$ & $<1.7 \times 10^{-7}$ \\
ZS 102 & 0.8 & & $<1.7 \times 10^{-5}$ \\
ZS 161 & 0.7 & & \\
M 348 & 0.7 & &
\end{tabular}

Note. Photometry used is from Stauffer et al. (2009) and references therein.

a $\log L_{X}<29.3$, and it accounts roughly for the drop-off in sensitivity with off-axis angle of the telescope.

Blanco 1. Photometry was obtained from Stauffer et al. (2009). Fractional luminosities were computed using the same method as for the Pleiades and NGC 2547 and are listed by star in Table 5. None of the 19 stars show an excess and a strong indicated stellar wind.

Including stars with upper limits, for all three samples it is uncommon for a star with a strong computed wind to show a significant infrared excess. Since only a modest wind is sufficient to dominate the grain removal (Minato et al. 2006; Plavchan et al. 2009), this result should be independent of the saturation effects. When we use the $\mathrm{K}-\mathrm{S}$ test, the lowest probability that this correlation arises by chance is about $12 \%$, using $F_{X} \sim 7.2 \times 10^{6} \mathrm{erg} \mathrm{s}^{-1} \mathrm{~cm}^{-2}$ as the threshold. As a check, we repeated this procedure using our upper limits on the fractional luminosities in place of the excess ratios at $24 \mu \mathrm{m}$ and found no evidence for a correlation. Allowing for the extra free parameter associated with adjusting the $\mathrm{K}-\mathrm{S}$ test to minimize the probability of a chance correlation, these results again suggest that there is no significant relation.

We also tried to estimate the level of saturation for the wind strengths, assuming that collisional timescales do not differ much from drag timescales (e.g., see Equation (A23) in Plavchan et al. 2009). A significant number of stars have substantial excesses, even though the indicated wind strength would produce substantial drag. It is therefore likely that the wind strengths are overestimated. However, we cannot say anything more quantitative due to the issues mentioned above as well as errors in our estimates of the fractional luminosities. More observations and/or an alternative estimate of wind strengths are needed to test the influence of stellar winds on infrared excesses.

\section{CONCLUSIONS}

We report the results of a new survey of solar-type stars in the Pleiades for $24 \mu \mathrm{m}$ excess indicative of circumstellar debris disks. We combine this work with previous surveys (Stauffer et al. 2005; Gorlova et al. 2006) to build a sample of 71 solar- type stars in this cluster with sufficiently accurate data to identify excesses as small as $10 \%$ at $24 \mu \mathrm{m}$. Twenty-three of these stars have excesses at this level or above; on statistical grounds, it is likely that about 6 additional members have excesses in the $6 \%-10 \%$ range, and the remaining 42 stars must have little or no $24 \mu \mathrm{m}$ excess. The incidence of excesses at $24 \mu \mathrm{m}$ and at the age of the Pleiades is high, $\sim 31 \% \pm 6 \%$.

We find that the incidence of $24 \mu \mathrm{m}$ excesses for solartype stars in the Pleiades is slightly smaller than for A stars (from a general sample mostly in the field; Su et al. 2006). It appears that by an age of $\sim 750 \mathrm{Myr}$, the excesses around solar-type stars have decayed faster than they decay around A stars (Gáspár et al. 2009). The effect probably arises through a mechanism that operates relatively slowly, such as systematic velocity differences in $24 \mu \mathrm{m}$ emitting zones of the debris disks around the two stellar types and the resulting difference in the speed of debris disk evolution.

Our study of the Pleiades, plus similar work on other clusters, lets us test aspects of debris disk behavior independently of the evolution of these systems with age. We confirm the results of Stauffer et al. (2009) that close, high-mass binary systems tend not to harbor debris disks. This behavior is probably associated with binary companions that orbit close to the zone where debris disks tend to lie (Trilling et al. 2007). There appear to be anti-correlations between infrared excesses and both rotation $(v \sin i)$ and X-ray luminosity, as also indicated by some previous works. However, we find that these results are not statistically significant and may arise instead from selection effects within the debris disk sample. The excesses around stars with indicated strong winds (from X-ray surface brightnesses) suggest that the wind strengths may be overestimated.

The authors thank András Gáspár for helpful discussions and the anonymous referee for comments which improved the paper. This research has made use of the SIMBAD database and the VizieR catalog access tool, operated at CDS, Strasbourg, France. This work also makes use of data products from the Two Micron All Sky Survey, which is a joint project of the University of Massachusetts and the Infrared Processing and Analysis Center/California Institute of Technology, funded by the National Aeronautics and Space Administration and the National Science Foundation. This work is also based, in part, on observations made with Spitzer Space Telescope, which is operated by the Jet Propulsion Laboratory, California Institute of Technology under NASA contract 1407 . This work supported by contract 1255094 from Caltech/JPL to the University of Arizona.

\section{REFERENCES}

Aigrain, S., Hodgkin, S., Irwin, J., Hebb, L., Irwin, M., Favata, F., Moraux, E., \& Pont, F. 2007, MNRAS, 375, 29

Alexander, R. 2008, New Astron. Rev., 52, 60

Allende Prieto, C., \& Lambert, D. L. 1999, A\&A, 352, 555

Andrews, S. M., \& Williams, J. P. 2005, ApJ, 631, 1134

Artyukhina, N. M., \& Kalinina, E. P. 1970, Trudy Sternberg, Astron. Inst., 40, 3 Balog, Z., et al. 2009, ApJ, 698, 1989

Belikov, A. N., et al. 2002, A\&A, 384, 145

Bessell, M. S. 1979, PASP, 91, 589

Boesgaard, A. M. 1989, ApJ, 336, 798

Boesgaard, A. M., Budge, K. G., \& Ramsay, M. E. 1988, ApJ, 327, 389

Boesgaard, A. M., \& Friel, E. D. 1990, ApJ, 351, 467

Bonatto, Ch., Bica, E., \& Girardi, L. 2004, A\&A, 415, 571

Bouvier, J., Rigaut, F., \& Nadeau, N. 1997, A\&A, 323, 139

Carpenter, J. M., Mamajek, E. E., Hillenbrand, L. A., \& Meyer, M. R. 2009a, ApJ, 705, 1646 
Carpenter, J. M., et al. 2008, ApJS, 179, 423

Carpenter, J. M., et al. 2009b, ApJS, 181, 197

Castelli, F., \& Kurucz, R. L. 2004, arXiv:astro-ph/0405087

Chen, C. H., Jura, M., Gordon, K. D., \& Blaylock, M. 2005, ApJ, 623, 493

Claria, J. J. 1982, A\&AS, 47, 323

Cranmer, S. R. 2008, in ASP Conf. Ser. 384, 14th Cambridge Workshop on Cool Stars, Stellar Systems, and the Sun, ed. G. van Belle (San Francisco, CA: ASP), 317

Currie, T., Plavchan, P., \& Kenyon, S. J. 2008, ApJ, 688, 597

Dominik, C., \& Decin, G. 2003, ApJ, 598, 626

Dullemond, C. P., Hollenbach, D., Kamp, I., \& D'Alessio, P. 2007, in Protostars and Planets V, ed. B. Reipurth, D. Jewitt, \& K. Keil (Tucson, AZ: Univ. Arizona Press), 555

Engelbracht, C. W., et al. 2007, PASP, 119, 994

Flesch, E., \& Hardcastle, M. J. 2004, A\&A, 427, 387

Ford, A., Jeffries, R. D., \& Smalley, B. 2005, MNRAS, 364, 272

Gáspár, A., et al. 2009, ApJ, 697, 1578

Glebocki, R., \& Stawikowski, A. 2000, Acta Astron., 50, 509

Gordon, K. D., et al. 2005, PASP, 117, 503

Gorlova, N., Rieke, G. H., Muzerolle, J., Stauffer, J. R., Siegler, N., Young, E. T., \& Stansberry, J. H. 2006, ApJ, 649, 1028

Gorlova, N., et al. 2007, ApJ, 670, 516

Gray, R. O., Graham, P. W., \& Hoyt, S. R. 2001, AJ, 121, 2159

Grigorieva, A., Artymowicz, P., \& Thébault, Ph. 2007, A\&A, 461, 537

Gustafson, B. A. S. 1994, Annu. Rev. Earth Planet. Sci., 22, 553

Habing, H. J., et al. 2001, A\&A, 365, 545

Hauck, B., \& Mermilliod, M. 1998, A\&AS, 129, 431

Hernández, J., et al. 2006, ApJ, 652, 472

Hertzsprung, E. 1947, Ann. Sternw. Leiden, 19, part I

Hillenbrand, L. A., et al. 2008, ApJ, 677, 630

Høg, E., et al. 2000, A\&A, 355, L27

Holzwarth, V., \& Jardine, M. 2007, A\&A, 463, 11

Iriarte, B. 1969, Bol. Obs. Tonantzintla y Tacubaya, 5, 89

Ivanov, G. A. 2008, Kinematika Fiz. Nebesnykh Tel., 24, 480

Jeffries, R. D., Evans, P. A., Pye, J. P., \& Briggs, K. R. 2006, MNRAS, 367, 781

Jeffries, R. D., \& Oliveira, J. M. 2005, MNRAS, 358, 13

Kähler, H. 1999, A\&A, 346, 67

Kharchenko, N. V., Piskunov, A. E., Röser, S., Schilbach, E., \& Scholz, R.-D. 2004, Astron. Nachr., 325, 740

Krist, J. E. 2006, Spitzer Tiny TIM User's Guide Version 2.0

Mamajek, E. E., \& Hillenbrand, L. A. 2008, ApJ, 687, 1264

Marigo, P., et al. 2008, A\&A, 482, 883

Marino, A. F., et al. 2008, A\&A, 490, 625

Martín, E. L., Dahm, S., \& Pavlenko, Y. 2001, in ASP Conf. Ser. 245, Astrophysical Ages and Times Scales, ed. T. von Hippel, C. Simpson, \& N. Manset (San Francisco, CA: ASP), 349

Mason, B. D., Hartkopf, W. I., McAlister, H. A., \& Sowell, J. R. 2003, AJ, 106, 637

Mayne, N. J., \& Naylor, T. 2008, MNRAS, 386, 261

Mendoza, E. E. 1967, Bol. Obs. Tonantzintla y Tacubaya, 4, 149

Mermilliod, J.-C., Mayor, M., \& Udry, S. 2009, A\&A, 498, 949

Mermilliod, J.-C., Rosvick, J. M., Duquennoy, A., \& Mayor, M. 1992, A\&A, 265,513
Meyer, M. R., et al. 2008, ApJ, 673, L181

Meynet, G., Mermilliod, J.-C., \& Maeder, A. 1993, A\&AS, 98, 477

Micela, G., et al. 1990, ApJ, 348, 557

Minato, T., Köhler, M., Kimura, H., Mann, I., \& Yamamoto, T. 2006, A\&A, 452,707

Monet, D. G., et al. 2003, AJ, 125, 984

Moshir, M., et al. 1990, BAAS, 22, 1325

Noyes, R. W., Hartmann, L. W., Baliunas, S. L., Duncan, D. K., \& Vaughan, A. H. 1984, ApJ, 279, 763

Panagi, P. M., \& O’Dell, M. A. 1997, A\&AS, 121, 213

Pels, G., Oort, J. H., \& Pels Kluyver, H. A. 1975, A\&A, 43, 423

Perryman, M. A. C., et al. 1997, A\&A, 323, L49

Philip, A. G. D., \& Egret, D. 1980, A\&AS, 40, 199

Plavchan, P., Jura, M., \& Lipscy, S. J. 2005, ApJ, 631, 1161

Plavchan, P., et al. 2009, ApJ, 698, 1068

Queloz, D., Allain, S., Mermilliod, J.-C., Bouvier, J., \& Mayor, M. 1998, A\&A, 335,183

Rebull, L. M., et al. 2008, ApJ, 681, 1484

Rieke, G. H., et al. 2004, ApJS, 154, 25

Rieke, G. H., et al. 2005, ApJ, 620, 1010

Rieke, G. H., et al. 2008, AJ, 135, 2245

Roeser, S., \& Bastian, U. 1988, A\&AS, 74, 449

Rosvick, J. M., Mermilliod, J.-C., \& Mayor, M. 1992, A\&A, 255, 130

Siegler, N., Muzerolle, J., Young, E. T., Rieke, G. H., Mamajek, E. E., Trilling, D. E., Gorlova, N., \& Su, K. Y. L. 2007, ApJ, 654, 580

Silverstone, M. D., et al. 2006, ApJ, 639, 1138

Skrutskie, M. F., et al. 2006, AJ, 131, 1163

Soderblom, D. R., Jones, B. F., Balachandran, S., Stauffer, J. R., Duncan, D. K., Fedele, S. B., \& Hudon, J. D. 1993, AJ, 106, 1059

Soderblom, D. R., Laskar, T., Valenti, J. A., Stauffer, J. R., \& Rebull, L. M 2009, AJ, 128, 1292

Spangler, C., Sargent, A. I., Silverstone, M. D., Becklin, E. E., \& Zuckerman, B. 2001, ApJ, 555, 932

Stauffer, J. R., \& Hartmann, L. W. 1987, ApJ, 318, 337

Stauffer, J. R., Schultz, G., \& Kirkpatrick, J. D. 1998, ApJ, 499, L199

Stauffer, J. R., et al. 2005, AJ, 130, 1834

Stauffer, J. R., et al. 2007, ApJS, 172, 663

Stauffer, J. R., et al. 2009, ApJ, submitted

Su, K. Y. L., et al. 2006, ApJ, 653, 675

Suchkov, A. A., Makarov, V. V., \& Voges, W. 2003, ApJ, 595, 1206

Tokunaga, A. T. 2000, in Allen's Astrophysical Quantities, ed. A. N. Cox (4th ed.; New York: Springer), 151

Trilling, D. E., et al. 2007, ApJ, 658, 1289

Trilling, D. E., et al. 2008, ApJ, 674, 1086

Van Leeuwen, F., Alphenaar, P., \& Brand, J. 1986, A\&AS, 65, 309

Westerlund, B. E., Garnier, R., Lundgren, K., Petterson, B., \& Breysacher, J. 1988, A\&AS, 76, 101

White, R. J., Gabor, J. M., \& Hillenbrand, L. A. 2007, AJ, 133, 2524

Wood, B. E., Müller, H.-R., Zank, G. P., Linsky, J. L., \& Redfield, S. 2005, ApJ, 628, L143

Wright, C. O., Egan, M. P., Kraemer, K. E., \& Price, S. D. 2003, AJ, 125, 359

Wyatt, M. C. 2005, A\&A, 433, 1007

Wyatt, M. C. 2008, ARA\&A, 46, 339 